Protoporphyrin zwischen gekreuzten Nicols rot aufleuchtet. Aus den genannten Umständen ergibt sich der Verdacht, daß es sich bei dem Augenpigment der Sagitten um einen porphyrinartigen Körper handelt. Ein Luminescenzmikroskop zur Prüfung des Fluorescenzverhaltens (nach Zugabe von Pyridin) stand mir nicht zur Verfügung. Es mag auch zweifelhaft erscheinen, ob man bei der geringen Menge der vorhandenen Substanz auf diesem Wege ans Ziel gelangt, wenigstens bei den kleinen Sagitten der Nordsee. Da aber andere Arten weit größere Augen mit erheblich mehr Pigment besitzen, so möchte ich Forschern, welche die Gelegenheit haben, derartiges Material frisch mit dem Fluorescenzmikroskop zu untersuchen, eine solche Prüfung nahelegen.

\section{Einfluß von Streptomycin auf Samen normaler und chlorophylldefekter Gerste}

\author{
Von Hans von Euler \\ Vitamin-Institut der Universität Stockholm \\ (Z. Naturforschg. 5 b, 448 [1950]; eingeg. am 29. Dez. 1950)
}

In einer vor 4 Jahren unternommenen Untersuchung 1 über die Keimung von Getreidekörnern wurde die folgende Beobachtung gemacht: Läßt man z. B. Gerstensamen nach 10-20-stdg. Quellung in Wasser $\left(+1 / 2 \% \mathrm{H}_{2} \mathrm{O}_{2}\right)$ in verdünnter Lösung von Streptomycin, Merck, Rahway (StRM) zwischen bzw. auf Filtrierpapier weiter keimen, so fallen 2 Erscheinungen auf, eine unspezifische und eine spezifische:

1. Die starke Verkürzung und Krümmung der Wurzeln, wie sie auch durch andere antibiotische Stoffe (z. B. Acridin, 5-Brom-uracil) hervorgerufen werden kann. Die Klauenform der Wurzeln beraubt den Pflanzen ihren festen Halt und erschwert also ihre natürliche, aufrechte Stellung.

2. Die Hemmung der Chlorophyllbildung: Schon bald nach Beginn der Keimung in Lösung von etwa $75 \gamma$ STRM per $\mathrm{ml}$ zeigen sich am 3.-5. Keimungstag die Koleoptile etwas verdickt und das daraus hervortretende Keimblatt ist nur im oberen Teil grün, der untere erweist sich als chlorophyllfrei und rein weiß. Das zweite Blatt ist entweder ganz weiß oder besitzt nur eine grüne Spitze, deren Länge je nach der STRM-Konzentration und der Quellungsdauer zwischen $5 \mathrm{~mm}$ und $50 \mathrm{~mm}$ variiert.

Die (nur in vivo erfolgende) chemische Antichlorophyllwirkung hat uns besonders interessiert wegen der Analogie mit der früher eingehend von uns studierten Mutation an chlorophylldefekten Gersten-Mutanten Nilsson-Ehles ${ }^{2}$. Wir hatten nachgewiesen, daß bei diesen chlorophylldefekten Mutanten der Katalase-Gehalt (die KatalaseWirkung) gleichzeitig mit dem Chlorophyllgehalt abnimmt, womit zum ersten Male eine Mendel-Spaltung für einen Enzymgehalt experimentell gezeigt worden war.

Unser Gerstenmaterial vom Albina-Typus mendelt in rezessive weiße und dominante grüne Mutanten $(2 / 4 \mathrm{He}$ terozygoten, 1/4 Homozygoten. Es war nun zu untersuchen, wie sich dieses Albina-Material gegen STRM verhält, ob also dessen Empfindlichkeit gegen STRM gegenüber der entsprechenden normalen Gerste verändert ist.

$\mathrm{Um} \mathrm{zu}$ einem deutlichen Ergebnis zu gelangen, muß man die Samen mit solchen STRM-Konzentrationen und nach solchen Quellungszeiten behandeln, daß wenigstens die Spitzen der Keimblätter noch deutlich grün bleiben; nach einer Keimungsdauer von 10 Tagen werden die (heterozygoten) Keimblätter 70-125 mm lang und die Spitzen wurden in einer Länge von 5-32 $\mathrm{mm}$ grün mit ziemlich scharfer Abgrenzung.

An 2 Versuchsreihen, A und B, waren die Ergebnisse mit je 80 Samen die folgenden:

\section{A}

$\begin{array}{lc}\text { Albina } 1 & 20 \text { weiße } \\ 75 \gamma \text { STRM } / \mathrm{ml} & 39 \text { teilw. grüne } \\ & \begin{array}{r}19 \text { grüne } \\ 2 \text { ungekeimt }\end{array} \\ & \\ \text { Albina } 1 & 18 \text { weiße } \\ \text { unbehandelt } & 59 \text { grüne } \\ & 3 \text { ungekeimt } \\ \text { Siegergerste } & 65 \text { teilw. grüne } \\ \text { Svalöv } & 14 \text { grüne } \\ 75 \gamma \mathrm{STRM} / \mathrm{ml} & 1 \text { ungekeimt }\end{array}$

Hypothesenfrei besagen diese Zahlen, daß die heterozygoten, grünen Pflanzen der untersuchten Albina-Mutanten auf den STRM-Einfluß wesentlich stärker reagieren als die entsprechende chlorophyllnormale Gerstensippe (Siegergerste, Svalöv).

Die Chlorophyllsynthese ist, den genetischen Untersuchungen zufolge, die Nilsson-Ehle und seine Mitarbeiter an verschiedenen chlorophyllmutierenden Gerstensippen angestellt haben, je von mehreren Erbfaktoren bedingt ${ }^{3}$. Im Gegensatz zu früheren Autoren haben wir stets angenommen, daß die den genetischen Chlorophylldefekten zugrunde liegenden Wirkungen.(Mutationen) in der Inaktivierung gewisser Komponenten der Chlorophyllsynthese bestehen.

Bei dem an den untersuchten Heterozygoten beobachteten STRM-Effekt superponiert sich eine chemisch bedingte Inaktivierung eines an der Chlorophyllsynthese beteiligten Enzyms über die an den unbehandelten Mutanten vorliegenden (genetisch bedingten) Einflüsse.

Die beiden enzymatischen Antichlorophyll-Wirkungen, die genetisch bedingte und die durch STRM hervorgerufene, sind von einer Katalase-Hemmung begleitet, und es liegt also nahe, in Betracht zu ziehen, daß die beiden sich superponierenden Wirkungen mit Vorgängen an der Porphyringruppe in Beziehung stehen.

1 H. v. Eu ler, Kem. Arbeten, Ny F. II, Bd. 9, Mai 1947; H. v. E u ler u. M. J a a r m a, Ark. Kem. Mineral. Geol. Ser. A 25, Nr. 7 [1947]; H. v. Eu ler, Ark. Kem. Mineral. Geol. Ser. A 26, Nr. 6 [1948]; H. v. Euler u. L. Heller, Ark. Kem. Mineral Geol. Ser. A 1, Nr. 35 [1949].

2 H. v. Euler u. D. Run eh jelm, Ark. Kem. Mineral. Geol. Ser. A 10, Nr. 10 [1930]; H. v. Eu ler, Hereditas 13, 61 [1929]; H. v. Euler, H. Hellström u. D. Burströ m, Hoppe-Seyler's Z. physiol. Chem. 218, 241 [1933].

Die Antichlorophyllwirkung des STRM wurde an der Flagellate Euglena gracilis bestätigt von L. Provasoli u. Mitarb., Proc. Soc. exp. Biol. Med. 69, 279 [1948].

3 Vgl. H. v. Eu le r, H. D a vid s on u. D. Runeh jelm, Hoppe-Seyler's Z. physiol. Chem. 190, 247 [1930]. 